\title{
Effect of Pharmacist-Driven Professional Continuous Glucose Monitoring in Adults with Uncontrolled Diabetes
}

\author{
Christina H. Sherrill, PharmD, BCACP, CPP; Christopher T. Houpt; \\ Elisabeth M. Dixon; and Scott J. Richter, PhD
}

\begin{abstract}
BACKGROUND: Diabetes requires close monitoring to achieve optimal outcomes and avoid adverse effects. Continuous glucose monitoring (CGM) is one approach to measuring glycemia and has become more widespread with recent advances in technology; however, ideal implementation of CGM into clinical practice is unknown. CGM can be categorized as personal CGM, which can be for at-home use to replace self-monitoring of blood glucose, or professional CGM (proCGM), which is used intermittently under the direction of a health care professional. The expanding role of the clinical pharmacist allows pharmacists to be at the forefront of implementing proCGM technology, but literature on the effect of pharmacist-driven proCGM is lacking. Pharmacists and physicians within 1 physician-owned clinic used proCGM technology differently. Pharmacists conducted 1 or 2 office visits to interpret data and make interventions, while physicians interpreted data 1 time and relayed interventions via phone.
\end{abstract}

OBJECTIVES: To (a) compare the change in hemoglobin A1c from baseline to 6 months between the different methods of proCGM implementation, and (b) describe and compare the clinical interventions made as a result of the different methods of proCGM implementation.

METHODS: In this retrospective cohort study, adults identified in the electronic medical record via Current Procedural Terminology code 95250 or 95251 undergoing proCGM with CGM data interpreted and baseline A1C $\geq 7 \%$ were included. Patients with additional CGM use within the 6 -month follow-up period were excluded. Data collection included demographics, A1c at baseline and during the 6-month follow-up period, and CGMassociated interventions. Patients were categorized as undergoing 1 pharmacist-driven encounter (RPh1), 2 pharmacist-driven encounters (RPh2), or 1 physician-driven encounter (MD1) for proCGM implementation. Combined RPh1 and RPh2 (cRPh) data were also used for analysis. The primary outcome was change in A1c from baseline to 6 months, which was evaluated by analysis of covariance.

RESULTS: Of 378 patient charts reviewed, 315 instances of proCGM implementation met inclusion criteria (58 RPh1, 35 RPh2, 222 MD1), and 253 had post-implementation A1c data for analysis of the primary outcome (52 RPh1, 30 RPh2, 171 MD1). Baseline A1c was $8.4 \%, 8.8 \%$, and $9.1 \%$ with mean reduction from baseline to 6 months of $1.0 \%, 1.3 \%$, and $0.6 \%$, respectively. cRPh patients experienced a greater mean reduction in A1c compared with MD1 $(P=0.002)$. RPh2 patients had a statistically significant reduction compared with MD1 $(P=0.005)$, but RPh1 patients did not $(P=0.054)$. The number of CGM-associated pharmacological interventions was 1.33 for RPh1 patients, 1.63 for RPh2 at the first encounter and 1.34 at the second, and 1.17 for MD1.

CONCLUSIONS: Pharmacist-driven implementation of proCGM was associated with greater A1c reductions and more pharmacological interventions versus physician-driven implementation. This study demonstrated improved clinical outcomes with pharmacists providing direct patient care through implementation of new diabetes technology.

J Manag Care Spec Pharm. 2020;26(5):600-09

Copyright $\odot 2020$, Academy of Managed Care Pharmacy. All rights reserved.

\section{What is already known about this subject}

Diabetes management is multifaceted and often requires close monitoring and frequent intervention to achieve desired outcomes and limit adverse effects.

Emerging technologies for diabetes management, including continuous glucose monitoring (CGM), are important tools that are changing the way clinicians care for patients with diabetes. There is no standard protocol for implementing professional CGM into clinical practice, and literature investigating pharmacist use of this technology is lacking

\section{What this study adds}

This article adds to the current body of literature by examining the use of professional CGM by clinical pharmacists and comparing pharmacist-driven implementation to physician-driven implementation.

This study reveals that pharmacist-driven implementation of professional CGM is associated with greater reductions in hemoglobin Alc and more clinical interventions than physician-driven implementation, thus demonstrating the value of pharmacists in providing this clinical service.

Results also suggest that 2 evaluations approximately 7 days apart of a single professional CGM device by a pharmacist is more effective at reducing Alc than 1 evaluation by a pharmacist, particularly in patients with insulin-dependent diabetes.

$\mathrm{D}$ iabetes mellitus affects more than 30 million Americans and cost the U.S. health care system $\$ 327$ billion in 2017. ${ }^{1}$ Diabetes is a complex chronic disease state that often requires close monitoring and frequent interventions to achieve optimal therapeutic control and avoid adverse effects. While hemoglobin Alc and self-monitoring of blood glucose (SMBG) have long been standards for measuring glycemic control, recent advances in technology for continuous glucose monitoring (CGM) have made this method of glycemic measurement more widespread and accessible. Furthermore, as of 2019, American Diabetes Association's Standards of Medical Care in Diabetes includes a new chapter dedicated to diabetes technology in which CGM and its place in therapy are discussed. ${ }^{2}$

CGM devices measure glucose through minimally invasive sensors in the interstitial fluid for 3 to 14 days. Sensors report 
interstitial glucose every 5 to 15 minutes, which has been found to correlate with capillary blood glucose. ${ }^{3}$ CGM devices may be divided into 2 types: real-time CGM and intermittently scanning, or "flash," CGM. Real-time CGM continuously reports glucose levels, while intermittently scanning CGM provides glucose data only when the user scans the device. ${ }^{2}$

CGM can also be categorized as personal use, where the patient uses CGM technology at home to supplement or replace SMBG with results available on demand, or professional use, where results are often blinded to the patient and only viewable in the health care setting. ${ }^{4}$ Personal CGM (perCGM) equipment is provided by a pharmacy or durable medical equipment provider and may cost the patient hundreds of dollars per month. ${ }^{5}$ Professional CGM (proCGM) is used intermittently under the direction of a health care professional and can be conducted during a regular office visit using Current Procedural Terminology (CPT) codes.

Various studies have demonstrated the benefit of perCGM, showing Alc reductions of $1.0 \%$ with perCGM plus SMBG versus $0.5 \%$ with SMBG alone, as well as reduction in hypoglycemia (43 minutes vs. 80 minutes in the control). ${ }^{6,7}$ Another study showed perCGM users to have a significantly lower number of severe hypoglycemic events (14 compared with 34 without perCGM). ${ }^{8}$ Furthermore, the amount of time spent in euglycemia can be increased to $73.7 \%$ with perCGM compared with $68.3 \%$ in the control. ${ }^{9}$

While these positive outcomes have been demonstrated with perCGM, literature describing the role of proCGM systems and their effects on diabetes management is more limited. Studies have found modest Alc decreases between $0.4 \%$ and $0.7 \%$ from baseline Alc. The greatest benefit has been seen in patients using multiple daily insulin injections or continuous subcutaneous insulin infusions. ${ }^{10,11}$

Additionally, literature on pharmacist implementation of this technology is particularly scarce, despite evidence that pharmacists' contributions in general diabetes team-based care can have a significant effect with an average Alc reduction of $1.1 \%$ compared with usual care. ${ }^{12}$ A small study found that pharmacist implementation of proCGM resulted in an Alc reduction from $9.0 \%$ to $8.3 \%$ after 6 months. ${ }^{13}$ There remains a critical need to investigate the role of the clinical pharmacist in proCGM implementation. Determining optimal use of this technology will help improve diabetes patient care and optimize use of resources.

In this study, the use of a proCGM system capable of recording glucose data for 14 days was analyzed. Pharmacists and physicians within 1 physician-owned clinic used this technology differently. Pharmacists conducted 1 or 2 office visits to interpret data and make interventions, while physicians interpreted data 1 time with interventions typically relayed via phone.
The purpose of this study was to determine which proCGM implementation method (1 interpretation encounter with a pharmacist, 2 interpretation encounters with a pharmacist, or 1 interpretation encounter with a physician) was most effective at improving diabetes outcomes and optimizing pharmacological interventions. The study objectives were to (a) compare the change in Alc from baseline to 6 months between the different methods of proCGM implementation, and (b) describe and compare the clinical interventions made as a result of the different methods of proCGM implementation.

\section{Methods}

\section{Device}

In this study, the FreeStyle Libre Pro (Abbott Diabetes Care, Alameda, CA) was used, which is a proCGM system approved by the U.S. Food and Drug Administration in September 2016. The FreeStyle Libre Pro system consists of single-user sensors and multiuser readers and was the first to not require calibration via glucometer readings. A sensor is placed on the back of a patient's upper arm with a filament inserted into the interstitial fluid to record glucose every 15 minutes for up to 14 days. Data can be retrieved multiple times during the sensor's 14-day duration of use by scanning the sensor with the reader.

\section{Setting and Clinical Procedures}

Patient care was conducted at a physician-owned primary care office with endocrinology, internal medicine, and clinical pharmacy services. Pharmacists in this setting worked under a collaborative practice agreement and could manage diabetes (including medication changes) after a referral from a physician within the practice. Referrals for diabetes management typically came from the internal medicine physicians, and patients were generally not seen by both a pharmacist and endocrinologist for diabetes care. Procedures for pharmacist use of proCGM technology at this facility have been reported elsewhere. ${ }^{14}$ In brief, clinical pharmacists could see patients for a set of 3 clinic visits per proCGM implementation. The first visit would include placing and activating the CGM sensor, while the second and third visits would involve scanning the sensor, analyzing the data, and making clinical interventions based on data from the single sensor. With the device's capability of collecting glucose data over a period of 14 days, the second visit would occur approximately 1 week after sensor placement with the third visit approximately 2 weeks after placement. Patients following this procedure with 2 pharmacist-driven encounters for CGM data interpretation were categorized as "RPh2" for the study.

While the intent of pharmacist-driven proCGM implementation was to follow the above procedures, some patients only had 1 encounter with the pharmacist for CGM data analysis for various reasons, such as time constraints, sensor loss, or patient loss to follow-up. Patients with 1 pharmacist-driven 


\section{FIGURE 1 Inclusion Flowchart}

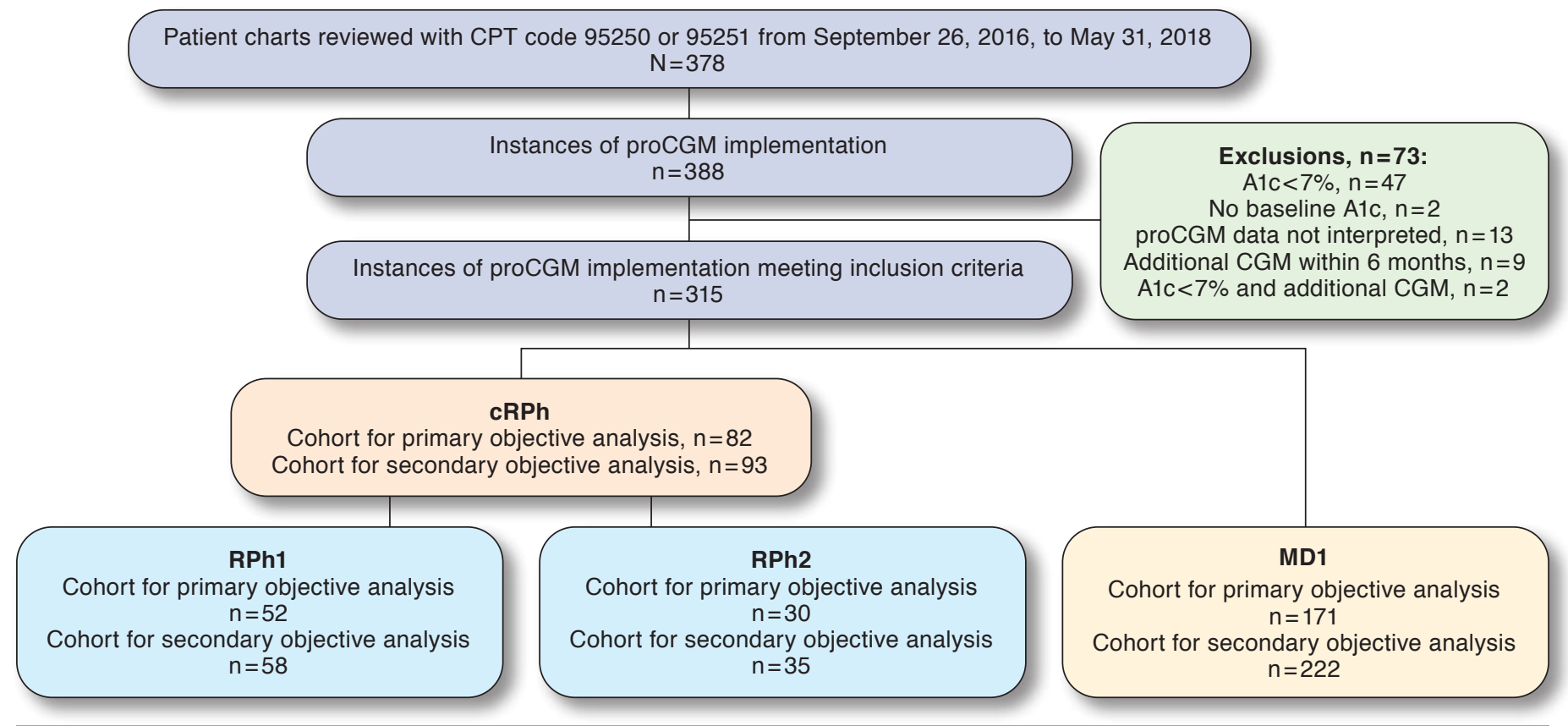

Note: All instances of proCGM implementation meeting inclusion criteria $(n=315)$ were included in the secondary objective analysis (clinical interventions). Only those instances with Alc values available during the 6-month follow-up period $(n=253)$ were included in the primary objective analyses (Alc change).

Alc=hemoglobin Alc; CGM = continuous glucose monitoring; CPT =Current Procedural Terminology; $c R P h=$ combined RPh1 and RPh2; MD1 = patients with 1 physiciandriven encounter; proCGM = professional continuous glucose monitoring; $R P h 1 / 2=$ patients with 1 or 2 pharmacist-driven encounters.

clinic visit for CGM data interpretation were categorized as "RPhl" for study procedures.

Physician-driven proCGM implementation typically consisted of an in-office encounter to place the CGM device, followed by the patient presenting to the office approximately 1 week later to have the CGM sensor scanned by a certified medical assistant. CGM data would then be shared with the physician, who would analyze the data and determine appropriate interventions. Results and interventions were communicated to the patient, usually via phone (95\% of patients), rather than an office visit. Patients with 1 physician-driven encounter for CGM data interpretation were categorized as "MDl" for study procedures.

\section{Study Design}

This single-center retrospective study evaluated Alc and clinical interventions resulting from pharmacist-driven and physician-driven proCGM implementation. Electronic medical records were queried for patients who had a CPT code 95250 or 95251 (CGM-specific billing codes) billed between September 26, 2016, and May 31, 2018, followed by a manual review to identify patients who were 18 years of age or older, had a baseline Alc of at least 7\%, and had proCGM implemented by a pharmacist or physician with data available for interpretation.
Patients with additional CGM use (whether personal or professional) within the 6-month follow-up period were excluded in an attempt to isolate the effects of a single CGM implementation method during the study period. Data collection included demographics, baseline Alc, CGM-associated interventions, and Alc and clinical encounters during the 6-month follow-up period. Baseline medications were considered those that the patient was taking before the CGM placement visit. CGMassociated interventions were those documented in the note corresponding to the proCGM data interpretation encounter. Patients were categorized as undergoing 1 pharmacist-driven encounter (RPh1), 2 pharmacist-driven encounters (RPh2), or 1 physician-driven encounter (MD1) for CGM data interpretation from a single sensor, as noted above. Combined RPhl and RPh2 (cRPh) data were also used for analysis.

Study data were collected and managed using Research Electronic Data Capture (REDCap), a secure, web-based application hosted at High Point University. ${ }^{15}$ Investigators reviewed the first 50 patient charts individually and compared findings to ensure consistency in data collection. Data were also reviewed for consistency and accuracy at the conclusion of data collection through the reporting feature in REDCap. This study was approved by the local institutional review board. 


\section{Outcomes}

The primary outcome was change in Alc from baseline to 6 months. Secondarily, clinical interventions documented for a proCGM interpretation encounter were recorded and categorized as pharmacological or nonpharmacological. Pharmacological interventions consisted of initiating, discontinuing, and changing medication therapy, including switching from 1 formulation to another (e.g., changing metformin immediate release to extended release) or shifting the timing of dosing (e.g., changing the timing of insulin glargine from bedtime to morning). Pharmacological interventions were also classified as increasing medication use (i.e., initiating a new medication or increasing the dose of an existing medication) or decreasing medication use. Patients could have multiple pharmacological interventions per encounter. Recorded nonpharmacological interventions included lifestyle interventions (diet, exercise, and weight loss) and patient education (hypoglycemia management, medication administration, and medication adherence).

\section{Data Analysis}

Data were de-identified before analysis. Bonferroni-adjusted 95\% confidence intervals were used to compare baseline characteristics between groups for patients with post-implementation Alc. Least squares regression was used to fit an analysis of covariance model to estimate and generate t-tests to compare mean reductions in Alc from baseline. The last Alc measurement within the 6-month follow-up period was the dependent variable. The independent variables were implementation type (cRPh, RPh1, RPh2, or MD1) and baseline Alc. Secondary analyses added insulin treatment (treated or not treated at baseline) along with baseline Alc measurement as covariates. Body mass index, type of diabetes, and total daily insulin dose were considered as additional covariates, but they were not associated with change in Alc and removed from the model. Statistical significance was defined as a $P$ value $<0.05$. All analyses were implemented using R statistical software (R Foundation for Statistical Computing, Vienna, Austria).

\section{Results}

A total of 315 instances of proCGM implementation were included, consisting of 308 individual patients, 7 of whom had an additional proCGM implementation more than 6 months after the initial proCGM implementation (Figure 1). Of the 315 included instances of proCGM implementation, 253 had Alc measurements during the follow-up period for analysis of the primary outcome. Of all included patients, most were female and white with diagnoses of type 2 diabetes mellitus, hypertension, and hyperlipidemia (Table 1). The mean age was 62.6 years with mean body mass index of $34.0 \mathrm{~kg}_{\text {per }} \mathrm{m}^{2}$ and baseline Alc of $9.0 \%$. Most patients were treated with metformin and insulin. Of those with documented doses of insulin, the mean total daily dose was 91.4 units. Bonferroni-adjusted 95\% confidence intervals showed no difference between groups, except for body mass index for patients in the RPh2 and MD groups. While all patients had baseline Alc of at least 7\%, hypoglycemia or intent of deprescribing was a documented indication for proCGM use in $12.1 \%$ of RPhl patients, $17.1 \%$ of $\mathrm{RPh} 2$, and $34.7 \%$ of $\mathrm{MD} 1$.

\section{Change in A1c}

Change in Alc was analyzed for the 253 instances of proCGM implementation that had Alc data within 6 months of proCGM placement. The average time from the proCGM placement visit to the Alc value used for analysis was 112 days for the RPhl cohort, 123 days for RPh2, and 124 days for MD1. Unadjusted mean Alc reductions from baseline to 6 months were 1.1\%, $1.0 \%, 1.3 \%$, and $0.6 \%$ for the cRPh, RPh1, RPh2, and MD1 cohorts, respectively, which were all statistically significant with $P<0.001$ (Table 2). Compared with MD1, patients in $c R P h$ and $\mathrm{RPh} 2$ both experienced a greater mean reduction in Alc $(P=0.002$ and $P=0.005$, respectively).

When adjusted for insulin treatment at baseline and stratified by treated versus not treated, statistically significant reductions in Alc from baseline to 6 months were seen across all groups (Table 2). When comparing between groups, adjusted results for all patients with post-implementation Alc showed statistical significance for $\mathrm{CRPh}$ to $\mathrm{MD} 1, \mathrm{RPh} 1$ to $\mathrm{MD}$, and $\mathrm{RPh} 2$ to MDl ( $P=0.002, P=0.045$, and $P=0.004$, respectively). Of note, 5 patients were included in the "not treated" cohort who were started on insulin therapy at their CGM placement visit (1 RPh2, $4 \mathrm{MDl}$ ). A total of 10 "not treated" patients were started on insulin by the end of the 6-month follow-up period (2 RPh1, 3 RPh2, $5 \mathrm{MD} 1$ ).

Comparing groups separately under the different insulin categories revealed no statistically significant differences between groups for patients not treated with insulin therapy (Table 2). Analyses for patients treated with insulin, however, did reveal statistically significant Alc reductions for $\mathrm{cRPh}$ versus MDl, RPh2 versus MDl, and RPh2 versus RPhl ( $P=0.002$, $P<0.001$, and $P=0.034$, respectively). Overall, patients who were not undergoing insulin treatment at baseline had a $0.5 \%$ greater Alc reduction, on average, than patients undergoing insulin treatment $(P=0.010)$, regardless of the classification of proCGM implementation.

The percentage of patients with Alc less than $7 \%$ by the end of the 6-month follow-up period was $42.3 \%$ for RPh1, 30.0\% for $\mathrm{RPh} 2$, and $12.9 \%$ for $\mathrm{MD} 1$.

\section{Clinical Interventions}

The number of pharmacological interventions made during proCGM interpretation encounters was 1.33 for RPhl patients, 1.63 for RPh2 patients at the first encounter and 1.34 at the second encounter, and 1.17 for MDl (Figure 2). Insulin was 


\begin{tabular}{|c|c|c|c|c|c|c|}
\hline \multirow[b]{3}{*}{$\overline{\text { Age, } \text { mean years (SD) }}$} & \multicolumn{3}{|c|}{ Overall Cohort $(n=315)$} & \multicolumn{3}{|c|}{ Patients with Post-Implementation Alc $(n=253)$} \\
\hline & RPhl $(n=58)$ & $\mathrm{RPh} 2(\mathrm{n}=35)$ & MD1 $(n=222)$ & RPhl $(\mathrm{n}=52)$ & $\mathrm{RPh} 2(\mathrm{n}=30)$ & MD1 $(n=171)$ \\
\hline & $64.1 \quad(12.7)$ & $65.8 \quad(9.5)$ & $61.7(12.0)$ & $63.4(12.4)$ & $66.6 \quad(8.4)$ & $62.8(11.5)$ \\
\hline Female, $\mathrm{n}(\%)$ & $32(55.2)$ & $19(54.3)$ & $153(68.9)$ & $28(53.8)$ & $16(53.3)$ & $122(71.3)$ \\
\hline \multicolumn{7}{|l|}{ Race, n (\%) } \\
\hline Asian & $1 \quad(1.7)$ & $0 \quad(0.0)$ & $4 \quad(1.8)$ & $1 \quad(1.9)$ & $0 \quad(0.0)$ & $3 \quad(1.8)$ \\
\hline African American & $18(31.0)$ & $6 \quad(17.1)$ & $71(32.0)$ & $15(28.8)$ & $6(20.0)$ & $60 \quad(35.1)$ \\
\hline White & $38(65.5)$ & $25(71.4)$ & $137(61.7)$ & $35(67.3)$ & $20(66.7)$ & $102(59.6)$ \\
\hline Hispanic & $1 \quad(1.7)$ & $0 \quad(0.0)$ & $3 \quad(1.4)$ & $1 \quad(1.9)$ & $0 \quad(0.0)$ & $2 \quad(1.2)$ \\
\hline Other & $(0.0)$ & $4(11.4)$ & (3.2) & $(0.0)$ & $4(13.3)$ & $(2.3)$ \\
\hline Unknown & $(0.0)$ & $0 \quad(0.0)$ & $(0.5)$ & $(0.0)$ & $0 \quad(0.0)$ & $(0.6)$ \\
\hline \multicolumn{7}{|l|}{ Ethnicity, n (\%) } \\
\hline Hispanic or Latino & $1 \quad(1.7)$ & $1 \quad(2.9)$ & $3 \quad(1.4)$ & $1 \quad(1.9)$ & $1 \quad(3.3)$ & $(1.2)$ \\
\hline Not Hispanic or Latino & $57(98.3)$ & $33(94.3)$ & $214(96.4)$ & $51 \quad(98.1)$ & $28(93.3)$ & $166(97.1)$ \\
\hline Unknown & $0 \quad(0.0)$ & $1 \quad(2.9)$ & $5 \quad(2.3)$ & $0 \quad(0.0)$ & $1 \quad(3.3)$ & $3 \quad(1.8)$ \\
\hline Alc, mean \% (SD) & $(1.5)$ & $(1.8)$ & $(1.6)$ & (1.4) & $(1.6)$ & $(1.6)$ \\
\hline Body mass index, mean $\mathrm{kg} / \mathrm{m}^{2}(\mathrm{SD})^{\mathrm{a}}$ & $34.7 \quad(6.7)$ & $30.9 \quad(5.9)$ & $34.3 \quad(7.8)$ & $34.7 \quad(6.9)$ & $30.3 \quad(5.6)$ & $34.6 \quad(7.7)$ \\
\hline \multicolumn{7}{|l|}{ Medical conditions, $\mathrm{n}(\%)$} \\
\hline Type 1 diabetes mellitus & $2 \quad(3.4)$ & $3(8.6)$ & $37(16.7)$ & $2 \quad(3.8)$ & $2 \quad(6.7)$ & $25(14.6)$ \\
\hline Type 2 diabetes mellitus & $56(96.6)$ & $31(88.6)$ & $185(83.3)$ & $50(96.2)$ & $27(90.0)$ & $146 \quad(85.4)$ \\
\hline Unspecified diabetes & $\begin{array}{ll}0 & (0.0) \\
\end{array}$ & $1 \quad(2.9)$ & $0 \quad(0.0)$ & $\begin{array}{ll}0 & (0.0) \\
\end{array}$ & $1 \quad(3.3)$ & $0 \quad(0.0)$ \\
\hline Hypertension & $52(89.7)$ & $29(82.9)$ & $198(89.2)$ & $46(88.5)$ & $24(80.0)$ & $155(90.6)$ \\
\hline Hyperlipidemia & $51 \quad(87.9)$ & $30(85.7)$ & $185(83.3)$ & $46(88.5)$ & $25(83.3)$ & $145(84.8)$ \\
\hline Coronary artery disease & $8(13.8)$ & $6 \quad(17.1)$ & $14 \quad(6.3)$ & $7(13.5)$ & $6(20.0)$ & $10 \quad(5.8)$ \\
\hline Myocardial infarction & $5 \quad(8.6)$ & $2 \quad(5.7)$ & $(0.0)$ & $5 \quad(9.6)$ & $2 \quad(6.7)$ & $\begin{array}{ll}0 & (0.0)\end{array}$ \\
\hline Cerebrovascular accident & $5 \quad(8.6)$ & $1 \quad(2.9)$ & $1 \quad(0.5)$ & $3 \quad(5.8)$ & $0 \quad(0.0)$ & $(0.0)$ \\
\hline Neuropathy & $8(13.8)$ & $4 \quad(11.4)$ & $81 \quad(36.5)$ & $7(13.5)$ & $4(13.3)$ & $54 \quad(31.6)$ \\
\hline Nephropathy & $20(34.5)$ & $10(28.6)$ & $81 \quad(36.5)$ & $16(30.8)$ & $8 \quad(26.7)$ & $60 \quad(35.1)$ \\
\hline Retinopathy & $1 \quad(1.7)$ & $2 \quad(5.7)$ & $27(12.2)$ & $\begin{array}{ll}1 & (1.9) \\
\end{array}$ & $1 \quad(3.3)$ & $19(11.1)$ \\
\hline \multicolumn{7}{|l|}{ Medications, n (\%) } \\
\hline Biguanide & $35(60.3)$ & $20 \quad(57.1)$ & $89(40.1)$ & $33(63.5)$ & $19(63.3)$ & $76 \quad(44.4)$ \\
\hline Sulfonylurea & $13(22.4)$ & $6 \quad(17.1)$ & $24(10.8)$ & $13(25.0)$ & $5(16.7)$ & $19(11.1)$ \\
\hline SGLT2 inhibitor & $8(13.8)$ & $6 \quad(17.1)$ & $19 \quad(8.6)$ & $8(15.4)$ & $6(20.0)$ & $15 \quad(8.8)$ \\
\hline DPP-IV inhibitor & $16(27.6)$ & $4 \quad(11.4)$ & $28(12.6)$ & $13(25.0)$ & $4(13.3)$ & $24(14.0)$ \\
\hline Thiazolidinedione & $1 \quad(1.7)$ & $3 \quad(8.6)$ & $1 \quad(0.5)$ & $1 \quad(1.9)$ & $3(10.0)$ & $1 \quad(0.6)$ \\
\hline GLP-1 receptor agonist & $9(15.5)$ & $7(20.0)$ & $11 \quad(5.0)$ & $9 \quad(17.3)$ & $6(20.0)$ & $11 \quad(6.4)$ \\
\hline Long-acting insulin & $23(39.7)$ & $20 \quad(57.1)$ & $95(42.8)$ & $21 \quad(40.4)$ & $18(60.0)$ & $72(42.1)$ \\
\hline Intermediate-acting insulin & $1 \quad(1.7)$ & $0 \quad(0.0)$ & $35(15.8)$ & $1 \quad(1.9)$ & $0 \quad(0.0)$ & $26(15.2)$ \\
\hline Short-acting insulin & $0 \quad(0.0)$ & $0 \quad(0.0)$ & $18 \quad(8.1)$ & $0 \quad(0.0)$ & $0 \quad(0.0)$ & $15 \quad(8.8)$ \\
\hline Rapid-acting insulin & $11 \quad(19.0)$ & $11 \quad(31.4)$ & $90(40.5)$ & $10 \quad(19.2)$ & $10 \quad(33.3)$ & $65(38.0)$ \\
\hline Mixed insulin & $3 \quad(5.2)$ & $1 \quad(2.9)$ & $62 \quad(27.9)$ & $3 \quad(5.8)$ & $1 \quad(3.3)$ & $47 \quad(27.5)$ \\
\hline Insulin pump & $0 \quad(0.0)$ & $2 \quad(5.7)$ & $11 \quad(5.0)$ & $0 \quad(0.0)$ & $1 \quad(3.3)$ & $9 \quad(5.3)$ \\
\hline Any insulin therapy, n (\%) & $26(44.8)$ & $24(68.6)$ & $205(92.3)$ & $24(46.2)$ & $20(66.7)$ & $155(90.6)$ \\
\hline Daily dose of basal insulin, mean units (SD) & $50.4(49.6)$ & $43.7(25.0)$ & $63.4(45.0)$ & $50.7(50.9)$ & $45.3(26.5)$ & $63.6(44.8)$ \\
\hline Daily dose of bolus insulin, mean units (SD) & $30.4(19.4)$ & $40.2(24.7)$ & $38.0(30.4)$ & $30.0(20.2)$ & $40.4(26.6)$ & $40.7(32.3)$ \\
\hline Total daily insulin dose, mean units (SD) & $68.2(58.0)$ & $65.3(43.0)$ & $97.7(68.2)$ & $68.4(60.5)$ & $69.7(45.9)$ & $99.9(68.4)$ \\
\hline
\end{tabular}

the medication class most commonly changed, diet was the most common lifestyle intervention, and hypoglycemia management was the most common patient education provided (Table 3). Further information can be found in Appendix A (available in online article). The percentage of patients experiencing any intervention was $93.1 \%, 100 \%$, and $94.1 \%$ for $\mathrm{RPh} 1$, RPh2 (combined for both encounters), and MD1, respectively (Appendix B, available in online article). The percentage with 


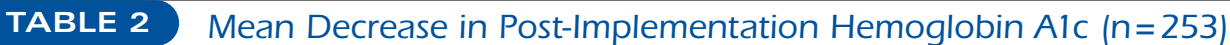

\begin{tabular}{|c|c|c|c|c|c|c|}
\hline & $\begin{array}{l}\text { Baseline Alc, } \\
\text { Mean \% (SD) }\end{array}$ & $\begin{array}{l}\text { Post-Implementation } \\
\text { Alc, Mean \% (SD) }\end{array}$ & $\begin{array}{c}\text { Mean \% Decrease in Alc } \\
(95 \% \mathrm{CI})\end{array}$ & & $\begin{array}{l}\text { tistic } \\
\text { f) }\end{array}$ & $P$ Value \\
\hline \multicolumn{7}{|c|}{ Unadjusted analysis } \\
\hline \multicolumn{7}{|c|}{ Baseline comparison by group } \\
\hline $\mathrm{cRPh}(\mathrm{n}=82)$ & $8.5 \quad(1.5)$ & $7.5 \quad(1.2)$ & $(0.8-1.4)$ & 7.35 & $(250)$ & $<0.001$ \\
\hline $\operatorname{RPhl}(\mathrm{n}=52)$ & $8.4 \quad(1.4)$ & $7.4 \quad(1.3)$ & $(0.6-1.3)$ & 5.17 & (250) & $<0.001$ \\
\hline $\mathrm{RPh} 2(\mathrm{n}=30)$ & $8.8(1.6)$ & $7.5(1.1)$ & $(0.8-1.8)$ & 5.30 & (250) & $<0.001$ \\
\hline MD1 $(n=171)$ & $9.1 \quad(1.6)$ & $8.5 \quad(1.7)$ & $(0.4-0.8)$ & 5.39 & (250) & $<0.001$ \\
\hline \multicolumn{7}{|c|}{ Comparison between groups } \\
\hline \multicolumn{3}{|c|}{ cRPh-MD1 } & $(0.2-0.9)$ & 3.13 & $(250)$ & 0.002 \\
\hline \multicolumn{3}{|l|}{ RPh2-MD1 } & $(0.2-1.3)$ & 2.81 & $(250)$ & 0.005 \\
\hline \multirow{2}{*}{\multicolumn{3}{|c|}{ RPhl-MDl }} & $(0.0-0.8)$ & 1.93 & (250) & 0.054 \\
\hline & & & $(-0.3-0.9)$ & 1.09 & (250) & 0.277 \\
\hline \multicolumn{7}{|c|}{$\begin{array}{l}\text { RPhL-RPnL } \\
\text { Adjusted for and stratified by insulin use }\end{array}$} \\
\hline \multicolumn{7}{|c|}{ Baseline comparison by group } \\
\hline \multicolumn{7}{|c|}{ All patients with post-implementation Alc $(n=253)$} \\
\hline \multicolumn{3}{|c|}{$\mathrm{cRPh}(\mathrm{n}=82)$} & $(1.0-1.6)$ & 9.10 & (248) & $<0.001$ \\
\hline \multicolumn{3}{|l|}{ RPhl $(n=52)$} & $(0.8-1.3)$ & 6.68 & (248) & $<0.001$ \\
\hline \multicolumn{3}{|l|}{$\operatorname{RPh} 2(n=30)$} & $(1.0-1.9)$ & 6.32 & (248) & $<0.001$ \\
\hline \multirow{2}{*}{\multicolumn{3}{|c|}{$\begin{array}{l}\text { MD1 }(n=171) \\
\text { Patients treated with insulin therapy }(n=199)\end{array}$}} & $(0.5-1.0)$ & 5.63 & (248) & $<0.001$ \\
\hline & & & & & & \\
\hline \multicolumn{3}{|c|}{$\operatorname{cRPh}(\mathrm{n}=44)$} & $(0.8-1.6)$ & 5.84 & (246) & $<0.001$ \\
\hline \multicolumn{3}{|l|}{ RPhl $(n=24)$} & $(0.3-1.3)$ & 3.12 & (246) & 0.002 \\
\hline \multicolumn{3}{|l|}{$\mathrm{RPh} 2(\mathrm{n}=20)$} & $(1.0-2.1)$ & 5.75 & (246) & $<0.001$ \\
\hline \multirow{2}{*}{\multicolumn{3}{|c|}{$\begin{array}{l}\text { MDl }(n=155) \\
\text { Patients not treated with insulin therapy }(n=54)\end{array}$}} & $(0.2-0.6)$ & 4.17 & (246) & $<0.001$ \\
\hline & & & & & & \\
\hline \multicolumn{3}{|c|}{$\begin{array}{l}\text { Patients not treated with insulin therapy }(\mathrm{n}=54) \\
\mathrm{cRPh}(\mathrm{n}=38)\end{array}$} & $(0.8-1.5)$ & 5.29 & (246) & $<0.001$ \\
\hline \multicolumn{3}{|l|}{ RPhl $(n=28)$} & $(1.0-2.0)$ & 6.46 & (246) & $<0.001$ \\
\hline \multicolumn{3}{|c|}{$\operatorname{RPh} 2(n=10)$} & $(0.1-1.7)$ & 2.34 & (246) & 0.020 \\
\hline \multirow{2}{*}{\multicolumn{3}{|c|}{$\begin{array}{l}\text { MD1 }(\mathrm{n}=16) \\
\text { Comparison between groups }\end{array}$}} & $(0.7-1.9)$ & 4.39 & (246) & $<0.001$ \\
\hline & \multicolumn{6}{|c|}{ Comparison between groups } \\
\hline All patients with pos & ntation Alc $(\mathrm{n}=$ & & & & & \\
\hline cRPh-MDl & & & $(0.2-0.9)$ & 3.14 & (248) & 0.002 \\
\hline RPh2-MD1 & & & $(0.2-1.2)$ & 2.90 & (248) & 0.004 \\
\hline RPhl-MDl & & & $0.4 \quad(0.0-0.9)$ & 2.02 & (248) & 0.045 \\
\hline RPh2-RPh1 & & & $0.3(-0.03-0.8)$ & 0.99 & (248) & 0.323 \\
\hline Patients treated with & erapy $(n=199)$ & & & & & \\
\hline cRPh-MDl & & & $(0.3-1.1)$ & 3.08 & (246) & 0.002 \\
\hline RPh2-MDI & & & $(0.6-1.7)$ & 4.00 & (246) & $<0.001$ \\
\hline RPhl-MDI & & & $(-0.2-0.9)$ & 1.37 & (246) & 0.171 \\
\hline RPh2-RPh1 & & & $(0.1-1.5)$ & 2.14 & (246) & 0.034 \\
\hline Patients not treated & $\mathrm{n}$ therapy $(\mathrm{n}=5$ & & & & & \\
\hline cRPh-MDl & & & $(-0.9-0.6)$ & -0.34 & (246) & 0.732 \\
\hline RPh2-MD1 & & & $(-1.4-0.5)$ & 0.87 & (246) & 0.383 \\
\hline RPhl-MDl & & & $(-0.6-0.9)$ & 0.44 & (246) & 0.660 \\
\hline RPh2-RPhl & & & $(-1.5-0.3)$ & -1.34 & (246) & 0.182 \\
\hline Insulin use & & & & & & \\
\hline Not treated-treated & & & $(0.1-1.0)$ & 2.59 & (248) & 0.010 \\
\hline
\end{tabular}

pharmacological interventions was $70.7 \%, 97.1 \%$, and $89.6 \%$, respectively, most of which represented increases in medication use.

\section{Discussion}

In this retrospective cohort study, patients undergoing pharmacist-driven and physician-driven proCGM implementation 


\section{FIGURE 2 Mean Number of Overall Interventions, Pharmacological Interventions, and Medication Classes Changed, Analyzed for All Patients Included in the Study for Each proCGM Interpretation Encounter $(n=315)$}

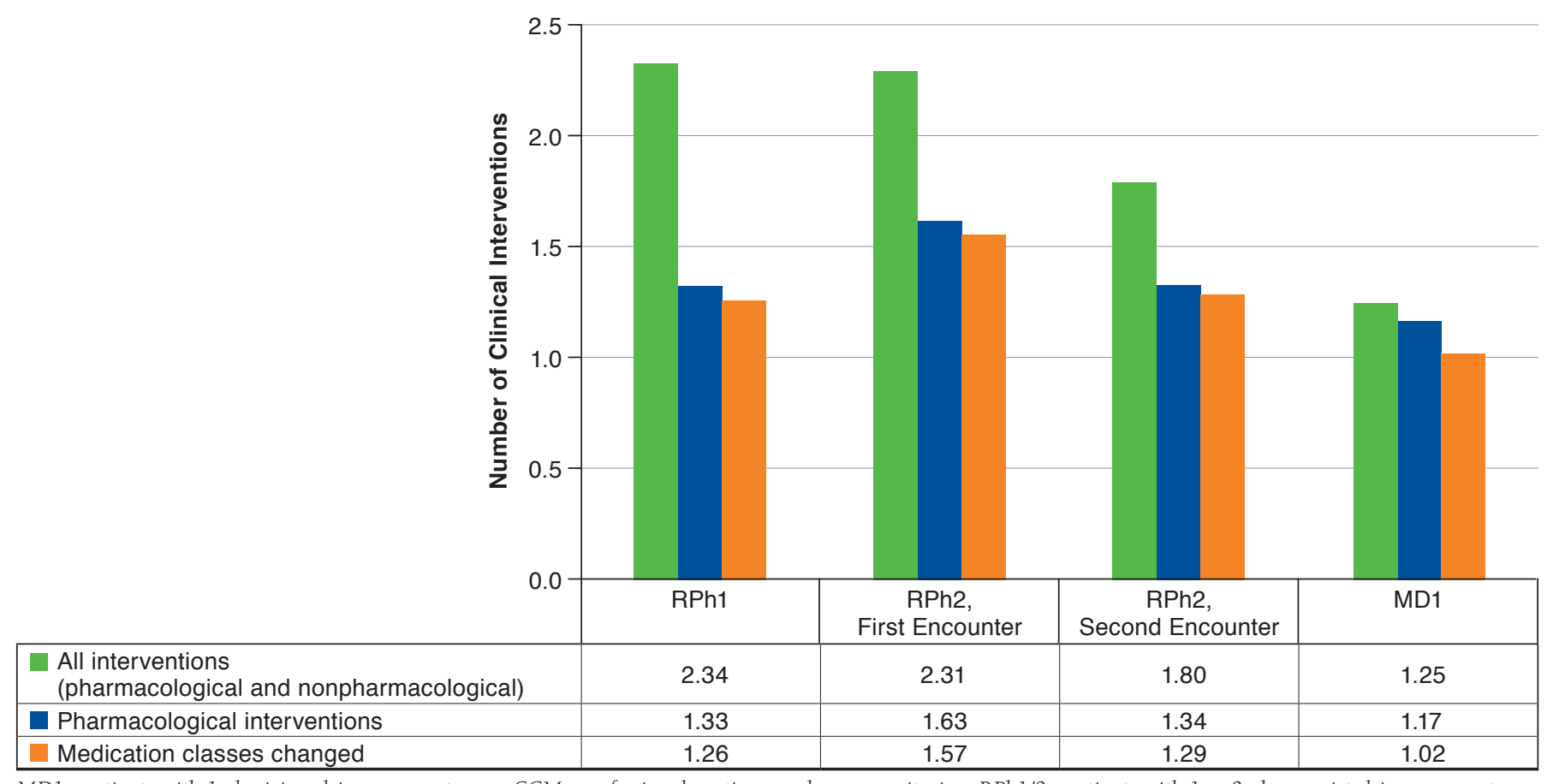

MDI = patients with 1 physician-driven encounter; proCGM=professional continuous glucose monitoring; RPh1/2=patients with 1 or 2 pharmacist-driven encounters.

experienced Alc reductions from baseline that were both clinically and statistically significant. Additionally, both unadjusted and adjusted analyses yielded statistically significantly greater reductions in the $\mathrm{CRPh}$ and $\mathrm{RPh} 2$ cohorts compared with physician-driven proCGM implementation. As the MDl cohort typically had results and interventions relayed via phone, results of this study raise the question of whether improved outcomes were because of the difference in the health care practitioner providing this service or the communication method. Furthermore, $34.7 \%$ of MDl patients had hypoglycemia or intent of deprescribing documented as an indication for proCGM use compared with $12.1 \%$ for the RPhl cohort and $17.1 \%$ for RPh2, which could have impacted the resultant Alc changes seen between groups. Lastly, the average number of diabetes-related encounters per patient after proCGM implementation during the 6-month follow-up period was 2.6 for RPh1 patients, 2.5 for RPh2, and 1.8 for MD1, suggesting that additional encounters and clinical interventions varied between groups and could have affected results.

While the cRPh and RPh2 cohorts had greater Alc reduction compared with MDl in both unadjusted and adjusted analyses, the reduction seen in the RPhl cohort was only statistically significantly better than MDl when adjusted for insulin therapy at baseline. The lack of difference in the unadjusted analysis may be interpreted as if the number of proCGM interpretation visits was the determining factor for seeing differences between groups. Alternatively, adjusting for insulin at baseline was an attempt to remove variance from insulin use and better determine factors that do have an effect. As such, the adjusted analysis suggests that pharmacist-driven proCGM implementation is more effective at improving Alc, even with the same number of encounters for CGM data interpretation.

Although the baseline comparison of Alc reduction achieved for the RPh2 cohort was greater than RPhl, the difference between groups was statistically significant only in the subpopulation of patients treated with insulin at baseline. A potential reason this difference is limited to the insulin-treated population is that the additional encounter in the RPh 2 cohort allowed for more fine-tuning of insulin therapy that may not be necessary or as impactful for noninsulin treatment options. Aside from directly comparing RPh2 to RPh1, it is noted that unadjusted analyses revealed that $\mathrm{RPh} 2$ but not $\mathrm{RPh} 1$ patients had a statistically significantly greater Alc reduction compared with MD1. While these data suggest that the clinical methods used for RPh2 patients were more effective at lowering Alc than those for RPhl for patients with insulin-dependent 
TABLE 3 Prevalence of Clinical Interventions Made During proCGM Interpretation Encounters ( $\mathrm{n}=315)$

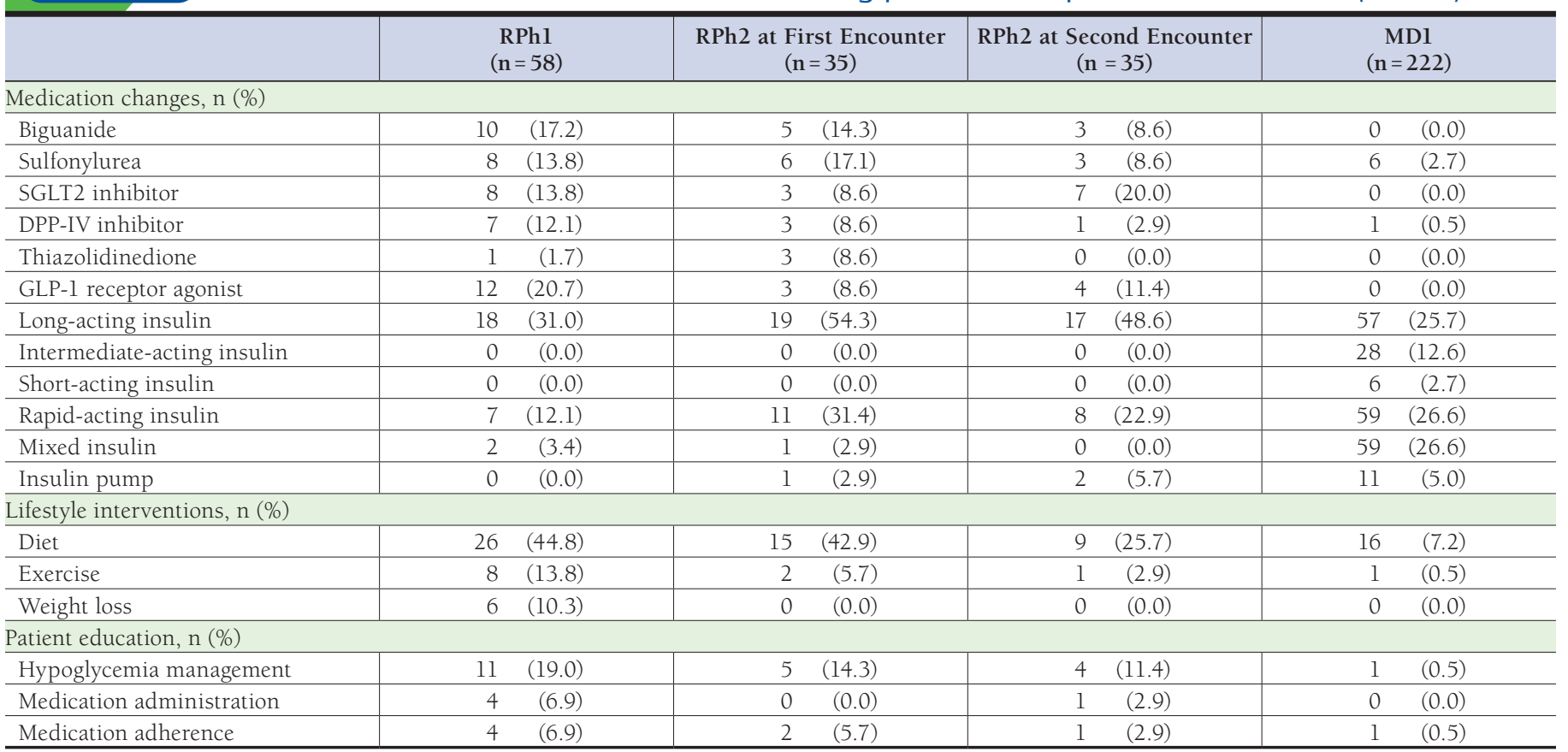

Note: Medication changes included any of the following: initiation, discontinuation, increase or decrease in dosage, change of formulation or delivery, or shift in administration time.

DPP-IV = dipeptidyl peptidase 4; GLP-1 = glucagon-like peptide-1; MDl = patients with 1 physician-driven encounter; proCGM=professional continuous glucose monitoring; RPh1/2 = patients with 1 or 2 pharmacist-driven encounters; SGLT2 = sodium-glucose cotransporter 2.

diabetes, a larger sample size is needed to further investigate whether a second office visit to interpret proCGM data is a costeffective use of resources to improve diabetes outcomes. As seen in Appendix B, only 88.6\% of RPh2 patients experienced any intervention at the second encounter compared with $93.1 \%$ for RPh1, 97.1\% for RPh2 first encounter, and 94.1\% for MD1. Still, more RPh2 patients experienced a pharmacological intervention at the second encounter compared with the number of pharmacological interventions at the first and only encounter for $\mathrm{RPhl}$ patients $(77.1 \%$ and $70.7 \%$, respectively).

More clinical interventions were made through pharmacist-driven than physician-driven proCGM implementation, though it is possible the communication method for the MDl interventions led to fewer interventions because of potential communication issues with messages being relayed via phone. Insulin was by far the most common type of medication changed, and interventions to increase medication use were more common than those to decrease medication use, which could be expected with the inclusion criteria of baseline Alc of at least $7 \%$. Data suggest an association between increased number of pharmacological interventions and greater Alc lowering across the 3 study groups.

Only 1 published study investigating the implementation of proCGM by clinical pharmacists was found. In this pre-/post- interventional study of 29 patients by Van Dril and Schumacher (2019), pharmacist-driven proCGM did not reveal a statistically significantly lower Alc compared with baseline. ${ }^{13}$ The authors noted that while not powered to detect a statistically significant difference, the observed $0.7 \%$ reduction in Alc was a "substantial improvement." A total of 54 pharmacological interventions were made across the 29 patients, averaging 1.86 interventions per patient. When compared with Van Dril and Schumacher, a greater decrease in mean Alc from baseline was observed in the current study, which did achieve statistical significance. A greater total number of pharmacological interventions was made in the RPh2 cohort (2.97 interventions for both encounters), but not for a single encounter for RPh2 or RPh1 patients. This study addresses a limitation acknowledged by Van Dril and Schumacher of lack of control group by comparing to physician-driven proCGM implementation. Demographics varied between the 2 studies, with the study by Van Dril and Schumacher consisting of mostly African American patients compared with the predominantly white population of the current study. The results of the current study build upon the limited literature in this area by expanding the sample size and patient population while comparing pharmacist-driven to physician-driven implementation. 
In addition to highlighting the pharmacist's role in implementing proCGM technology, this study also adds to the literature by including patients not using insulin at baseline to manage diabetes. While some data exist exploring the use of real-time CGM in patients on noninsulin therapy, literature on proCGM and intermittently scanning CGM for patients with noninsulin-dependent diabetes is lacking.,16 Although only approximately one fifth of study patients were not treated with insulin therapy at baseline, this study showed benefit of proCGM use in noninsulin-dependent patients across all study groups. Furthermore, patients not treated with insulin experienced even greater Alc lowering than those on insulin therapy at baseline. While duration of disease was not evaluated in this study, the aforementioned finding may be because of patients having early disease, less insulin resistance, and more room for clinical intervention and effect. Nonetheless, this study demonstrates that the use of proCGM technology can span the spectrum of diabetes care and that glucose data obtained from proCGM use in patients not treated with insulin may present an opportunity to guide clinical decisions regarding initiating and titrating medications and determining if and when insulin therapy should be introduced. Moreover, since Medicare requires patients to be insulin-dependent with 3 or more insulin injections per day for coverage of perCGM, proCGM allows patients who may not otherwise be eligible for this technology to benefit from the advances of CGM through professional-use devices.

Although not a focus of this article, it is important to acknowledge that the use of proCGM provides an avenue for pharmacists to bill for their services and generate revenue. The study by Van Dril and Schumacher cited above found that the mean payment was $\$ 126.87$ for use of CPT code 95250 and $\$ 39.17$ for 95251 (2017). ${ }^{13}$ The reimbursement potential combined with improved clinical outcomes demonstrates the benefit of pharmacist-driven proCGM implementation in improving patient care while promoting clinical pharmacy services.

By allowing pharmacists to participate in diabetes management with the use of proCGM technology, several of the goals of managed care can be achieved. Specifically, improved clinical outcomes were observed via decreases in Alc for patients seen by pharmacists. There is the potential for cost savings for patients and the health care system overall, as these improved clinical outcomes may lead to fewer hospitalizations and emergency room visits as well as the prevention or delay of advanced diabetes-related complications. Lastly, quality and accessibility of health care are achieved because pharmacists may be more accessible to patients to have face-to-face encounters as opposed to phone interventions.

\section{Limitations}

There are several limitations present in this study. The retrospective nature makes it susceptible to inconsistencies in clinical procedures or documentation of interventions, as well as potential bias and confounding based on patient inclusion in 1 study group versus another. The single-center design may limit generalizability as only a small number of practitioners were compared within 1 facility. The patient population included in this study may also affect generalizability, as patients were mostly white, whereas, the highest prevalence of diabetes is seen in the American Indian/Alaska Native, Hispanic, and non-Hispanic black populations. ${ }^{17}$ Additionally, other variables besides the health care professional providing the service may have affected the results of this study, such as the potential for longer or more frequent encounters for $\mathrm{cRPh}$ patients compared with MDl patients, including differences in the number of diabetes-related follow-up encounters after proCGM implementation. The type of encounter also has the potential to affect the results, as communicating results via phone in the MDl group may have affected patients' understanding of their results and the perceived importance of the recommended interventions. Furthermore, there is potential bias in that more patients in the MDl group had a documented indication for proCGM implementation of hypoglycemia or deprescribing than RPhl or RPh2 patients.

\section{Future Directions}

Several needs still exist in the realm of CGM research. A well-designed prospective multicenter trial could improve generalizability and internal validity. A prospective study with pharmacists relaying proCGM results and interventions via phone may help answer the question of whether improved Alc as seen in this study was because of the type of practitioner or communication method. This could also be addressed by having physicians provide office visits to interpret proCGM data and make interventions. As this study did not reach statistical significance when directly comparing $\mathrm{RPh} 1$ to $\mathrm{RPh} 2$ for all patients, further research should be done to determine if a 2-step pharmacist-driven proCGM implementation leads to better outcomes and additional meaningful interventions compared with a l-step pharmacist-driven proCGM implementation. While this study uncovered interesting patterns in insulin-treated versus nontreated patients, additional studies are needed to further elucidate the role of proCGM in patients not treated with insulin. Lastly, as this study excluded patients with baseline Alc below 7\%, future studies could explore the pharmacist's role in using proCGM technology to deprescribe medications and reduce hypoglycemia in patients with baseline Alc less than $7 \%$.

\section{Conclusions}

Despite recent major advances in CGM technology, literature on the optimal implementation method of these devices is lacking. This study demonstrated that pharmacist-driven implementation of proCGM with 2 planned in-person follow-up 
visits was associated with greater reductions in Alc and more clinical interventions compared with physician-driven implementation with 1 follow-up encounter via phone. This study also showed that patients not treated with insulin therapy can benefit from proCGM use. As the role of the clinical pharmacist continues to expand, proCGM affords an opportunity for pharmacists to contribute to high levels of patient care while improving diabetes outcomes.

\section{Authors}

CHRISTINA H. SHERRILL, PharmD, BCACP, CPP; ELISABETH M. DIXON, PharmD candidate; and CHRISTOPHER T. HOUPT, PharmD candidate, High Point University Fred Wilson School of Pharmacy, High Point, North Carolina. SCOTT J. RICHTER, PhD, University of North Carolina at Greensboro.

AUTHOR CORRESPONDENCE: Christina H. Sherrill, PharmD, BCACP, CPP, High Point University Fred Wilson School of Pharmacy, One University Pkwy, High Point, NC 27268. Tel.: 336.841.9678; E-mail: csherril@highpoint.edu.
3. Bailey T, Bode BW, Christiansen MP, Klaff LJ, Alva S. The performance and usability of a factory-calibrated flash glucose monitoring system. Diabetes Technol Ther. 2015;17(11):787-94.

4. Carlson AL, Mullen DM, Bergenstal RM. Clinical use of glucose monitoring in adults with type 2 diabetes. Diabetes Technol Ther. 2017;19(Suppl 2): S4-11.

5. Medtronic for Healthcare Professionals. Continuous glucose monitoring: the costs. Available at: https://hcp.medtronic-diabetes.com.au/cgm-subscriptions. Accessed February 18, 2020.

6. Beck RW, Riddlesworth T, Ruedy K, et al. Effect of continuous glucose monitoring on glycemic control in adults with type 1 diabetes using insulin injections: The DIAMOND randomized clinical trial. JAMA. 2017;317:371-78

7. Ehrhardt NM, Chellappa M, Walker MS, Fonda SJ, Vigersky RA. The effect of real-time continuous glucose monitoring on glycemic control in patients with type 2 diabetes mellitus. J Diabetes Sci Technol. 2011;5(3):668-75.

8. Van Beers CA, DeVries JH, Kleijer SJ, et al. Continuous glucose monitoring for patients with type 1 diabetes and impaired awareness of hypoglycaemia (IN CONTROL): a randomised, open-label, crossover trial. Lancet Diabetes Endocrinol. 2016;4(11):893-902.

9. Hermanns N, Schumann B, Kulzer B, Haak T. The impact of continuous glucose monitoring on low interstitial glucose values and low blood glucose values assessed by point-of-care blood glucose meters: results of a crossover trial. J Diabetes Sci Technol. 2014;8(3):516-522

10. Leinung M, Nardacci E, Patel N, et al. Benefits of short-term professional continuous glucose monitoring in clinical practice. Diabetes Technol Ther. 2013;15(9):744-47.

11. Kesavadev J, Vigersky R, Shin J, et al. Assessing the therapeutic utility of professional continuous glucose monitoring in type 2 diabetes across various therapies: a retrospective evaluation. Adv Ther. 2017;34(8):1918-27.

12. Fazel MT, Bagalagel A, Lee JK, Martin JR, Slack MK. Impact of diabetes care by pharmacists as part of health care team in ambulatory settings: a systematic review and meta-analysis. Ann Pharmacother. 2017;51(10):890-907.

13. Van Dril E, Schumacher C. Impact of professional continuous glucose monitoring by clinical pharmacists in an ambulatory care setting. J Am Coll Clin Pharm. 2019;2(6):638-44.

14. Sherrill $\mathrm{CH}$, Martin CM. Professional continuous glucose monitoring: 14-day procedure. In: Castelli G. ACCP Ambulatory Care Pharmacist's Survival Guide. 4th ed. Lenexa, KS: American College of Clinical Pharmacy; 2019:232-34.

15. Harris PA, Taylor R, Thielke R, Payne J, Gonzalez N, Conde JG. Research electronic data capture (REDCap) - a metadata-driven methodology and workflow process for providing translational research informatics support. J Biomed Inform. 2009;42(2):377-81.

16. Yoo HJ, An HG, Park SY, et al. Use of a real time continuous glucose monitoring system as a motivational device for poorly controlled type 2 diabetes. Diabetes Res Clin Pract. 2008;82(1):73-79.

17. Centers for Disease Control and Prevention. National diabetes statistics report, 2020. Available at: https://www.cdc.gov/diabetes/data/statistics/ statistics-report.html. Accessed April 3, 2020. 
APPENDIX A Raw Number of Medication Changes by Class and Study Cohort ( $\mathrm{n}=315)$

\begin{tabular}{|c|c|c|c|c|c|c|c|c|c|}
\hline & $\begin{array}{c}\mathrm{RPhl} \\
(\mathrm{n}=58)\end{array}$ & $\begin{array}{c}\mathrm{RPh} 2 \\
\text { at First } \\
\text { Encounter } \\
(\mathrm{n}=35)\end{array}$ & $\begin{array}{l}\mathrm{RPh} 2 \text { at } \\
\text { Second } \\
\text { Encounter } \\
(\mathrm{n}=35)\end{array}$ & $\begin{array}{c}\text { MD1 } \\
(\mathbf{n}=222)\end{array}$ & & $\begin{array}{c}\mathrm{RPhl} \\
(\mathrm{n}=58)\end{array}$ & $\begin{array}{c}\mathrm{RPh} 2 \\
\text { at First } \\
\text { Encounter } \\
(\mathrm{n}=35)\end{array}$ & $\begin{array}{l}\mathrm{RPh} 2 \text { at } \\
\text { Second } \\
\text { Encounter } \\
(\mathrm{n}=35)\end{array}$ & $\begin{array}{c}\text { MD1 } \\
(n=222)\end{array}$ \\
\hline \multicolumn{5}{|l|}{ Biguanide } & \multicolumn{5}{|l|}{ Long-acting insulin } \\
\hline Initiation & 3 & 0 & 0 & 0 & Initiation & 3 & 3 & 0 & 0 \\
\hline Increase dosage & 0 & 3 & 2 & 0 & Increase dosage & 8 & 11 & 9 & 47 \\
\hline Discontinuation & 2 & 0 & 0 & 0 & Discontinuation & 0 & 0 & 1 & 0 \\
\hline Decrease dosage & 3 & 0 & 0 & 0 & Decrease dosage & 5 & 5 & 6 & 14 \\
\hline \multicolumn{5}{|l|}{ Sulfonylurea } & \multicolumn{5}{|c|}{ Intermediate-acting insulin } \\
\hline Initiation & 0 & 3 & 0 & 1 & Initiation & 0 & 0 & 0 & 0 \\
\hline Increase dosage & 2 & 1 & 0 & 5 & Increase dosage & 0 & 0 & 0 & 24 \\
\hline Discontinuation & 4 & 2 & 0 & 0 & Discontinuation & 0 & 0 & 0 & 1 \\
\hline Decrease dosage & 1 & 0 & 2 & 0 & Decrease dosage & 0 & 0 & 0 & 12 \\
\hline \multicolumn{5}{|l|}{ SGLT2 inhibitor } & \multicolumn{5}{|l|}{ Short-acting insulin } \\
\hline Initiation & 7 & 2 & 3 & 0 & Initiation & 0 & 0 & 0 & 0 \\
\hline Increase dosage & 0 & 1 & 3 & 0 & Increase dosage & 0 & 0 & 0 & 5 \\
\hline Discontinuation & 1 & 0 & 0 & 0 & Discontinuation & 0 & 0 & 0 & 0 \\
\hline Decrease dosage & 0 & 0 & 0 & 0 & Decrease dosage & 0 & 0 & 0 & 1 \\
\hline \multicolumn{5}{|l|}{ DPP-IV inhibitor } & \multicolumn{5}{|l|}{ Rapid-acting insulin } \\
\hline Initiation & 0 & 0 & 0 & 1 & Initiation & 1 & 2 & 0 & 1 \\
\hline Increase dosage & 0 & 0 & 1 & 0 & Increase dosage & 2 & 7 & 4 & 52 \\
\hline Discontinuation & 7 & 3 & 0 & 0 & Discontinuation & 4 & 2 & 0 & 0 \\
\hline Decrease dosage & 0 & 0 & 0 & 0 & Decrease dosage & 0 & 1 & 5 & 5 \\
\hline \multicolumn{5}{|l|}{ Thiazolidinedione } & \multicolumn{5}{|l|}{ Mixed insulin } \\
\hline Initiation & 0 & 0 & 0 & 0 & Initiation & 0 & 0 & 0 & 2 \\
\hline Increase dosage & 0 & 0 & 0 & 0 & Increase dosage & 0 & 0 & 0 & 57 \\
\hline Discontinuation & 0 & 3 & 0 & 0 & Discontinuation & 2 & 1 & 0 & 1 \\
\hline Decrease dosage & 1 & 0 & 0 & 0 & Decrease dosage & 0 & 0 & 0 & 13 \\
\hline \multicolumn{5}{|c|}{ GLP-1 receptor agonist } & \multicolumn{5}{|l|}{ Insulin pump } \\
\hline Initiation & 12 & 2 & 3 & 0 & Initiation & 0 & 0 & 0 & 0 \\
\hline Increase dosage & 0 & 0 & 0 & 0 & Increase dosage & 0 & 1 & 0 & 11 \\
\hline Discontinuation & 0 & 0 & 1 & 0 & Discontinuation & 0 & 0 & 0 & 0 \\
\hline Decrease dosage & 0 & 1 & 0 & 0 & Decrease dosage & 0 & 0 & 0 & 2 \\
\hline
\end{tabular}

DPP-IV = dipeptidyl peptidase 4; GLP-1 = glucagon-like peptide-1; MDI = patients with 1 physician-driven encounter; proCGM= professional continuous glucose monitoring; RPh1/2 = patients with 1 or 2 pharmacist-driven encounters; SGLT2 = sodium-glucose cotransporter 2 . 
APPENDIX B Percentage of Patients with Any Intervention, Pharmacological Intervention, Increase in Medication Use, and Decrease in Medication Use, Analyzed for All Patients Included in the Study for Each proCGM Interpretation Encounter $(n=315)$

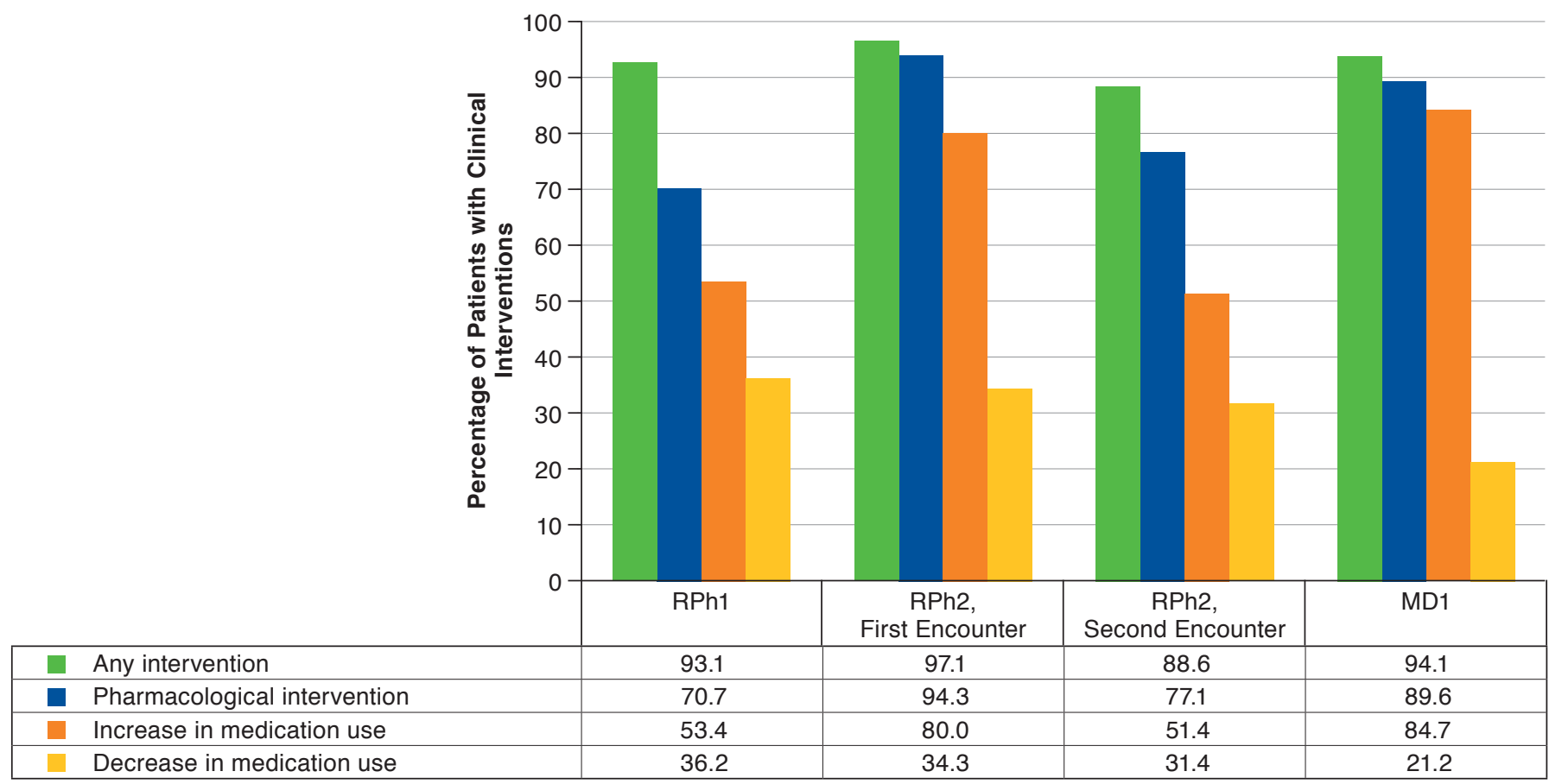

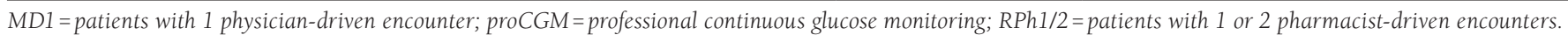

